\title{
Nonstationary nonlinear effects in optical microspheres
}

\section{Aleksey E. Fomin, Michael L. Gorodetsky, Ivan S. Grudinin, Vladimir S. Ilchenko}

Aleksey E. Fomin, Michael L. Gorodetsky, Ivan S. Grudinin, Vladimir S. Ilchenko, "Nonstationary nonlinear effects in optical microspheres," Proc. SPIE 5333, Laser Resonators and Beam Control VII, (1 June 2004); doi: $10.1117 / 12.530657$

Event: Lasers and Applications in Science and Engineering, 2004, San Jose, $\mathrm{Ca}$, United States 


\title{
Nonstationary nonlinear effects in optical microspheres
}

\author{
A.E. Fomin ${ }^{a}$, M.L. Gorodetsky ${ }^{a}$, I.S. Grudinin ${ }^{b}$, V.S. Ilchenko ${ }^{c}$ \\ ${ }^{a}$ Faculty of Physics, Moscow State University, 119992, Moscow, Russia; \\ ${ }^{b}$ California Institute of Technology, 103-33, Pasadena, CA 91125; \\ ${ }^{c}$ Jet Propulsion Laboratory, California Institute of Technology, 4800, Oak Grove Dr, Pasadena, \\ CA 91109-8099;
}

\begin{abstract}
Thermal nonlinearity can produce oscillatory instability in optical microspheres. We analyze theoretically the conditions of observations of this regime and demonstrate it experimentally. The observed curves are well compared with results of numerical modelling. In pure fused silica with low absorption thermal oscillations are suppressed due to concurrency with Kerr nonlinearity. We also describe for the first time experimentally observed slow and irreversible thermooptical processes in microspheres.
\end{abstract}

Keywords: Optical resonators, microspheres, nonlinear optics, thermal effects, chaos

\section{INTRODUCTION}

Optical microspheres with whispering gallery modes (WGMs) of total internal reflection ${ }^{1}$ as well as their novel toroidal derivatives ${ }^{2,3}$ uniquely combine small sub-millimeter size with volume of field localization $\left(V_{\text {eff }} \sim 10^{-9}\right.$ $\mathrm{cm}^{3}$ ) and very high quality factor. This combination provides, even for such "linear" material as fused silica, low threshold of optical bistability at microwatts of optical power, which scales like $V_{\text {eff }} / Q^{2}$. This nonlinear effect may be welcomed in some applications, and is undesirable in others. In addition to "fast" Kerr nonlinearity (by this term we denote the summary effect of cubic nonlinearities - due to electronic, strictional and lattice unharmonicity, inseparable in many cases), optical microspheres can exhibit "slow" thermal nonlinearity. This one results from the heating of mode volume by the power absorbed in a microsphere due to nonzero optical losses. The value of thermal nonlinearity is not constant but depends on the speed of internal intensity variations and therefore, on the history and rate of dynamic detuning of resonator modes from instantaneous input laser frequency. Basically, it can be described by two characteristic times. First is relevant to the thermal relaxation of the mode volume in the bulk of microsphere; the second one is associated with the thermal relaxation of microsphere as a whole. ${ }^{4}$ In the same paper (4), an interesting regime of oscillatory instability was observed (Fig.1). In Fig.1, representing a plot of intracavity intensity as a function of slowly changing input laser frequency, nonstationary relaxation oscillations are observed.

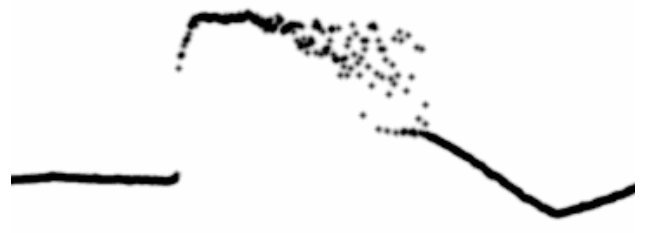

Figure 1. Optical nonlinear resonance and oscillatory instability in optical microsphere

Tentative explanation of this phenomenon, proposed in the article (4) required either two nonlinear mechanisms with different signs, or two excited modes in the resonator. It is known, however, that relaxation-type

I.S.G.: grudinin@caltech.edu 
nonlinearity itself can produce oscillatory instability even in a single mode resonator. ${ }^{5}$ The first attempt to explain the oscillatory instabilities in microspheres using this approach was made in the work of G.V. Belokopytov, ${ }^{6}$ however, quantitative results were in significant disagreement with the experiment.

Surprisingly, oscillatory and chaotic regimes sporadically observed in our early experiments, were never observed in later experiments with microspheres, both in our group and in other laboratories. The obvious reason, as we realized it recently, was that in subsequent research, the experiments were done with high-purity glasses in which predominant mechanism of loss, in the visible and near-infrared band, was scattering and not absorption. (Progress in our resonator fabrication technique allowed ${ }^{7}$ to reach fundamental limit of $Q \sim 10^{10}$ at $\lambda=0.63 \mu \mathrm{m}$.) As the theoretical analysis below shows, if the absorption is small enough for the thermalrelaxation nonlinearity to equal the instant Kerr one, competition of the two effects prevents the observation of oscillatory instability. As soon as we returned to fabrication of microspheres out of original, higher absorption silica, we were able to reproduce the effect and study it in detail.

\section{OPTICAL NONLINEARITY IN MICROSPHERES}

Wave equation for the electric field $\vec{E}_{s}$ in the sphere in first order approximation has the following form:

$$
\Delta \vec{E}_{s}-\frac{\epsilon_{s}(\vec{r})}{c^{2}}\left(\frac{\partial^{2} \vec{E}_{s}}{\partial t^{2}}+2 \delta \frac{\partial \vec{E}_{s}}{\partial t}\right)=\frac{4 \pi}{c^{2}}\left(\frac{\partial^{2} \vec{P}_{p}(\omega)}{\partial t^{2}}+\frac{\partial^{2} \vec{P}_{n}(\omega)}{\partial t^{2}}\right)
$$

where $\epsilon(\vec{r})$ is linear dielectric constant of the medium (equal to squared refractive index $n$ inside the sphere and unity outside), $\delta=\delta_{a}+\delta_{c}+\delta_{s}$ describes total losses, taking into account internal absorbtion, internal and external scattering and coupling losses $(Q=\omega / 2 \delta)$. The right part of the equation describes additional polarization due to pumping and due to nonlinear effects $\vec{P}_{n}=\chi_{n} \vec{E}_{s}$. Because of absorption, part of electromagnetic energy in the mode of the resonator transforms in thermal form and heats the resonator. Due to thermal dependence of refraction index this change of temperature will lead to the change of refractive index proportional to $u=T-T_{0}$. Accounting instant Kerr and this thermal nonlinearity we can write down $\chi$ as follows:

$$
\chi_{n}=\frac{\epsilon}{2 \pi} \beta\left(T-T_{0}\right)+\chi^{(3)}(\omega) E_{s}^{2},
$$

where $\beta=\frac{1}{n} \frac{\partial n}{\partial T}$ determines thermal dependence of the index of refraction. Using rotation wave approximation

$$
\vec{E}(\vec{r}, t)=a(t) \vec{E}_{0}(\vec{r}) e^{i \omega t}
$$

where $\vec{E}_{0}(\vec{r})$ describes normalized field distribution of a chosen eigenmode of the unperturbed lossless microsphere

$$
\int\left|\vec{E}_{0}\right|^{2} d \vec{r}=1
$$

and $a(t)$ is slowly varying amplitude, from the wave equation by multiplying it by $\vec{E}_{0}^{*}(\vec{r})$ and integrating over the whole volume we obtain the first nonlinear differential equation for our analysis:

$$
\dot{a}+a\left(\delta+\imath\left[\Delta \omega+\frac{2 \pi \omega_{0} \chi^{(3)}}{n^{2} V_{e f f}}|a|^{2}+\omega_{0} \beta \theta\right]\right)=\imath \sqrt{\frac{\pi \omega_{0} W}{n^{2} V_{e f f} Q}} .
$$

Here $\Delta \omega=\omega-\omega_{0}$ is detuning of the pumping frequency from the unperturbed resonance eigenfrequency $\omega_{0}, W$ is pump power, $\theta$ is the average heating over the volume of the mode:

$$
\theta=\int\left(T-T_{0}\right)\left|\vec{E}_{0}(\vec{r})\right|^{2} d \vec{r}
$$

and $V_{\text {eff }}$ is determined by the following simple formula

$$
V_{e f f}^{-1}=\int\left|\vec{E}_{0}(\vec{r})\right|^{4} d \vec{r} .
$$


Definition of effective volume (7) is very useful and appears in the same form not only for the problems of nonlinearity but also in analysis of scattering. ${ }^{8}$ It is not difficult to understand - to find the effective volume we should average the intensity distribution over this distribution, hence the fourth power. This definition is more formal and often more convenient than the alternative one: $\int\left|E_{0}\right|^{2} d v=\left|E_{0}\right|_{\text {max }}^{2} V_{\text {eff,m }}$, especially for complex modes with many maxima. For the most localized $T E_{\ell \ell 1}$ mode in microspheres $V_{\text {eff }} \simeq 2 V_{\text {eff }, m} \simeq$ $3.9 R^{11 / 6}(\lambda / n)^{7 / 6}$, where $R$ is radius of the microsphere.

\section{THERMAL EFFECT}

To calculate the effect of thermal nonlinearity we start with the equation of thermal diffusion:

$$
\frac{\partial T}{\partial t}-D \Delta T=\frac{n \alpha_{a} c}{4 \pi C \rho}|\vec{E}|^{2},
$$

where $D=\lambda^{*} / \rho C$ is thermal diffusivity, $\lambda^{*}$ is thermal conductivity, $\rho$ is density of the material, $C$ is specific heat capacity of the material of the resonator, $\alpha_{a}$ is part of coefficient of extinction due to absorption, $c$ is speed of light. Note that another component of losses - scattering does not influence thermal nonlinearity. $|\vec{E}|^{2}=I(t)\left|\vec{E}_{0}\right|^{2}$, where $I(t)=|a(t)|^{2}$ is slowly varying (as compared to optical relaxation time of the mode $\left.\frac{1}{I} \frac{d I}{d t} \ll \frac{\omega_{0}}{Q}\right)$ intensity. The density of energy in the mode is: $\mathcal{E}(t)=I(t) \frac{n^{2}}{4 \pi} V_{\text {eff }}$.

The relaxation of mode volume in the "infinite" media of microsphere may be found in spectral form as follows:

$$
T(t, \vec{r})-T_{0}=\frac{n \alpha_{a} c}{4 \pi C \rho} \frac{1}{(2 \pi)^{4}} \iint \frac{I(\Omega) G(\vec{q})}{D q^{2}+i \Omega} e^{i \Omega t+i \vec{q} \vec{r}} d \Omega d \vec{q},
$$

where

$$
G(\vec{q})=\int\left|\vec{E}_{0}\right|^{2} e^{-i \vec{q} \vec{r}} d \vec{r}
$$

With this approach thermal nonlinearity of the second kind connected with thermal relaxation of the microsphere itself is lost. For more rigorous analysis, decomposition in series of eigenfunctions with account of boundary conditions should be used. It is appropriate to note here that for the microsphere $100 \mu \mathrm{m}$ in diameter, the effective volume of the mode is nearly three orders of magnitude smaller than total volume of the sphere.

We are interested in the value $\theta(t)$ which is the average temperature difference over the volume of the mode:

$$
\theta(t)=\int\left(T-T_{0}\right)\left|\vec{E}_{0}(\vec{r})\right|^{2} d \vec{r}=\frac{n \alpha_{a} c}{4 \pi C \rho} \frac{1}{(2 \pi)^{4}} \iint \frac{I(\Omega)|G(\vec{q})|^{2}}{D q^{2}+i \Omega} e^{i \Omega t} d \vec{q} d \Omega=\frac{1}{2 \pi} \int \theta(\Omega) e^{i \Omega t} d \Omega .
$$

We can rather formally obtain the following equation for $\theta(\Omega)$ :

$$
i \Omega \theta(\Omega)+\delta_{\theta} \theta(\Omega)=\frac{n \alpha_{a} c}{4 \pi C \rho V_{\text {eff }}} I(\Omega),
$$

where

$$
\delta_{\theta}(\Omega)=\frac{D}{V_{e f f}}\left[\int \frac{|G(\vec{q})|^{2}}{q^{2}+i \Omega / D} \frac{d \vec{q}}{(2 \pi)^{3}}\right]^{-1}-i \Omega
$$

Expression (13) is hard to use and evaluate. However, in the range of frequencies where $\delta(\Omega)$ weakly depends on $\Omega$, reasonable estimate may be obtained in most cases directly from (8): $\Delta u \sim-2 u / b^{2}$, where $b$ is the half-thickness of the mode in the direction of largest gradient, which is radial direction for the microsphere. Hence

$$
\delta_{\theta} \simeq 2 D / b^{2}
$$


and the second differential equation for analysis is:

$$
\frac{\partial \theta}{\partial t}+\delta_{\theta} \theta=\frac{n \alpha_{a} c}{4 \pi C \rho V_{e f f}}|a(t)|^{2} .
$$

The value $1 / \delta_{\theta}$ has physical meaning of characteristic thermal relaxation time. The equations (5) and (13) lead to the nonlinear system of differential equations:

$$
\left\{\begin{array}{c}
\dot{u}+\delta u-\left(\Delta \omega+\mu\left(u^{2}+v^{2}\right)+\omega_{0} \beta \theta\right) v=0 \\
\dot{v}+\delta v+\left(\Delta \omega+\mu\left(u^{2}+v^{2}\right)+\omega_{0} \beta \theta\right) u=F \\
\dot{\theta}+\delta_{\theta} \theta=\frac{\nu \delta_{\theta}}{\omega_{0} \beta}\left(u^{2}+v^{2}\right)
\end{array}\right.
$$

where complex amplitude $a(t)$ from (5) was replaced by a sum of real quadrature components: $a(t)=u(t)+\imath v(t)$,

$\mu=\frac{2 \pi \omega_{0} \chi^{(3)}}{n^{2} V e f f}, \nu=\frac{\omega_{0} \beta n \alpha_{a} c}{4 \pi C \rho V_{e f f} \delta_{\theta}} F=\sqrt{\frac{\pi \omega_{0} W}{n^{2} V_{e f f} Q}}$. The system of equations (16) describes dynamical behavior of microresonator which takes into account nonlinear thermal effect and Kerr nonlinearity. Stationary solution of (16) is determined by the following cubic equation for the internal intensity $I_{0}=\left|a_{0}\right|^{2}=u^{2}+v^{2}$ :

$$
\left(\delta^{2}+\left(\Delta \omega+(\mu+\nu) I_{0}\right)^{2}\right) I_{0}-F^{2}=0 .
$$

This equation describes classical hysteretic response of the nonlinear resonator with two stable branches and one unstable branch. Intensity is maximal not at the resonance frequency but for the detuning $\Delta \omega=-(\mu+\nu) I_{0}$ produced both by Kerr and thermal effects. Nonlinear effects are pronounced and hysteresis is observable if this detuning is significantly larger than half-width of "linear" resonant curve $\delta$. Thermal nonlinearity dominates if $\nu>\mu$, i.e.

$$
\chi_{\theta}=\frac{n^{3} \alpha_{a} \beta c}{8 \pi^{2} C \rho \delta_{\theta}}>\chi^{(3)} .
$$

However, this equation is not enough to describe the behavior of the resonator for time intervals smaller than $1 / \delta_{\theta}$. Before going into analysis of dynamics of the system it is instructive to compare absolute values of thermal and Kerr effects in microspheres. In fused silica $\rho=2.2 \mathrm{~g} / \mathrm{cm}^{3}, C=6.7 \times 10^{6} \mathrm{erg} /(\mathrm{g} \cdot \mathrm{K}), \lambda^{*}=1.4 \times 10^{5} \mathrm{erg} /(\mathrm{cm} \cdot \mathrm{s} \cdot \mathrm{K})$, $D=9.5 \times 10^{-3} \mathrm{~cm}^{2} / \mathrm{s}, \beta=1 \times 10^{-5} \mathrm{~K}^{-1}$. For the fundamental mode $T E_{\ell \ell 1}$ in microsphere of medium size (index $\ell \simeq 2 \pi n R / \lambda=1000$, wavelength $\lambda=0.63 \mu \mathrm{m}, n=1.46$, radius $R=70 \mu \mathrm{m}$ ), radial width of the mode $b=0.84 R \ell^{-2 / 3} \simeq 0.6 \mu \mathrm{m}$. In this way we obtain from (14) $\delta_{\theta} \simeq 5 \times 10^{6} \mathrm{~s}^{-1}$. Fundamental absorption in fused silica for visible and near IR may be described ${ }^{9}$ as $\alpha_{a} \simeq 1.1 \times 10^{-3}[\mathrm{~dB} / \mathrm{km}] \exp [56 \mu \mathrm{m} / \lambda]$ which leads to $\alpha_{a} \simeq 4 \times 10^{-6} \mathrm{~cm}^{-1}$. This ultimately small absorption leads to $\chi_{\theta} \simeq 4 \times 10^{-16}$ esu which is nearly an order smaller than electronic $\chi^{(3)} \simeq 5 \times 10^{-15}$ esu. However, if real fused silica with impurities is used with $\alpha_{a} \simeq 6 \times 10^{-4} \mathrm{~cm}^{-1}$ corresponding to $Q=2 \pi n / \alpha \lambda=3 \times 10^{8}$, observed in early experiments, $\chi_{\theta} \simeq 6 \times 10^{-14}$ esu will be more than an order larger than Kerr nonlinearity. Therefore below we analyze separately two cases: a) the appearance of oscillatory instability due to only thermal nonlinearity and b) what happens with this instability when Kerr's nonlinearity appears.

\section{NONLINEAR REGIMES}

Dynamical behavior of the phase trajectories may be described by linearized equations obtained from (16) for the new set of small parameters $\xi=u-u_{0}, \eta=v-v_{0}, \zeta=\theta-\theta_{0}$ in the vicinity of equilibrium states $\left(u_{0}, v_{0}, \theta_{0}\right)$.

$$
\left\{\begin{array}{c}
\dot{\xi}=-\left(\delta-2 \mu u_{0} v_{0}\right) \xi+\left(\Delta_{n}+2 \mu v_{0}^{2}\right) \eta+\omega_{0} \beta v_{0} \zeta \\
\dot{\eta}=-\left(\Delta_{n}+2 \mu u_{0}^{2}\right) \xi-\left(\delta+2 \mu u_{0} v_{0}\right) \eta-\omega_{0} \beta u_{0} \zeta \\
\dot{\zeta}=2 \frac{\nu \delta_{\theta}}{\omega_{0} \beta} u_{0} \xi+2 \frac{\nu \delta_{\theta}}{\omega_{0} \beta} v_{0} \eta-\delta_{\theta} \zeta
\end{array}\right.
$$

where $\Delta_{n}=\Delta \Omega+(\nu+\mu) I_{0}=\Delta \Omega+(\nu+\mu) I_{0}$ is stationary nonlinear detuning from resonance. The solutions of equations (19) is determined by the roots of characteristic equation:

$$
\begin{gathered}
\lambda^{3}+\left(2 \delta+\delta_{\theta}\right) \lambda^{2}+\left((\mu+\nu)(3 \mu+\nu) I_{0}^{2}+2 \Delta \omega(2 \mu+\nu) I_{0}+\Delta \omega^{2}+\delta^{2}+2 \delta \delta_{\theta}\right) \lambda \\
+\delta_{\theta}\left(3(\mu+\nu)^{2} I_{0}^{2}+4 \Delta \omega(\mu+\nu) I_{0}+\Delta \omega^{2}+\delta^{2}\right)=0
\end{gathered}
$$






Figure 2. Areas of stability for nonlinear resonance with relaxational nonlinearity

From this algebraic equation, one may find areas of stability for the system (16) using Routh-Hurwitz criterion. The number of positive roots, leading to instabilities is determined by the number of sign changes in the sequence of $T_{0}=a_{0}, T_{1}=a_{1}, T_{1} T_{2}=\left(a_{2} a_{1}-a_{3}\right) T_{1}, a_{3}$, where $a_{0}, a_{1}, a_{2}, a_{3}$ are coefficients of equal powers of $\lambda$ in the polynomial (20). As $a_{0}=1$ and $a_{1}=2 \delta+\delta_{\theta}$ are positive, the behavior of the system is determined by the sequence of roots of the two other terms $T_{2}$ and $a_{3}$ :

$$
\begin{aligned}
I_{a 3} & =-\frac{2 \Delta \omega \pm \sqrt{\Delta \omega^{2}-3 \delta^{2}}}{3(\mu+\nu)} \\
I_{T 2} & =\frac{-\Delta \omega\left(2 \mu+\nu-\nu \delta_{\theta} / 2 \delta\right) \pm \sqrt{\Delta \omega^{2}\left(\mu-\nu \delta_{\theta} / 2 \delta\right)^{2}-\left(\delta+\delta_{\theta}\right)^{2}(\mu+\nu)\left(3 \mu+\nu-\nu \delta_{\theta} / \delta\right)}}{(\mu+\nu)\left(3 \mu+\nu-\nu \delta_{\theta} / \delta\right)} .
\end{aligned}
$$

The first pair gives the borders of unstable region of hysteretic resonance curve which is unobservable. The second pair shows the possibility of thermal oscillations which are of the main interest in this paper. These oscillations are possible if the interval determined by the second pair lies out of the borders of the first pair (Fig. 2).

The solutions (21) are greatly simplified in strong nonlinear regimes when $\nu I, \mu I,|\Delta \omega| \gg \delta, \delta_{\theta}$. In this case the zeros are given by three asymptotic straight lines: $I_{a 3,1}(\mu+\nu)=-\Delta \omega / 3, I_{a 3,2}(\mu+\nu)=I_{T 2,1}(\mu+\nu)=-\Delta \omega$, $I_{T 2,2}\left(3 \mu+\nu-\nu \delta_{\theta} / \delta\right)=-\Delta \omega$. The second asymptote coincides also with the asymptote for the maximum internal intensity $I_{0}$ for the nonlinear resonance curve. Thermal oscillations are possible only when $I_{T 2,2}>I_{a 3,2}$ i.e. $\nu>2 \mu \delta / \delta_{\theta}$ :

$$
\chi^{(3)}<\frac{\beta n^{4}}{8 \pi^{2} C \rho} \frac{\alpha_{a}}{\alpha_{\Sigma}} \simeq 4 \times 10^{-14} \frac{\alpha_{a}}{\alpha_{\Sigma}} \text { esu, }
$$

which means that Kerr nonlinearity suppresses thermal oscillations in pure fused silica where quality factor is mostly determined by scattering and $\alpha_{a} \ll \alpha_{\Sigma}$. Here $\alpha_{\Sigma}=\alpha_{a}+\alpha_{n a}=2 \pi n / \lambda Q$ describes total losses in the microsphere which include not only internal absorption, but internal scattering, losses on surface inhomogeneities and in adsorbed surface layers as well as additional losses due to the optical coupler.

\section{NUMERICAL MODELLING}

Numerical modelling of the dynamical behavior of the system described by equations (16), was performed in MATLAB package (C)The MathWorks, Inc. for the realistic set of parameters of microspheres observed in experiments. The Runge-Kutta method of 4 th an 5 th order was used. The obtained results of numerical modelling are in complete accordance with analytical calculations and are in agreement with observed experimental response curves. As it is clearly seen on Fig.3 for the specific set of parameters the oscillatory instability is observed on one of the slopes of the nonlinear resonant curve. This curve is close to that observed in (1), which was similar to Fig. 1. 
Stable focus-type equilibrium state in the interval determined by zeroes of $T_{2}$ loses stability and soft bifurcation of Andronov-Hopf type with ultimate cycle is formed (Fig.3). For numerical and analytical evaluations the following parameters, the same as for Fig.2, were used: input power of $150 \mu \mathrm{W}$ and microsphere diameter of $100 \mu \mathrm{m}$ with TE WGM having $l-m=20$.
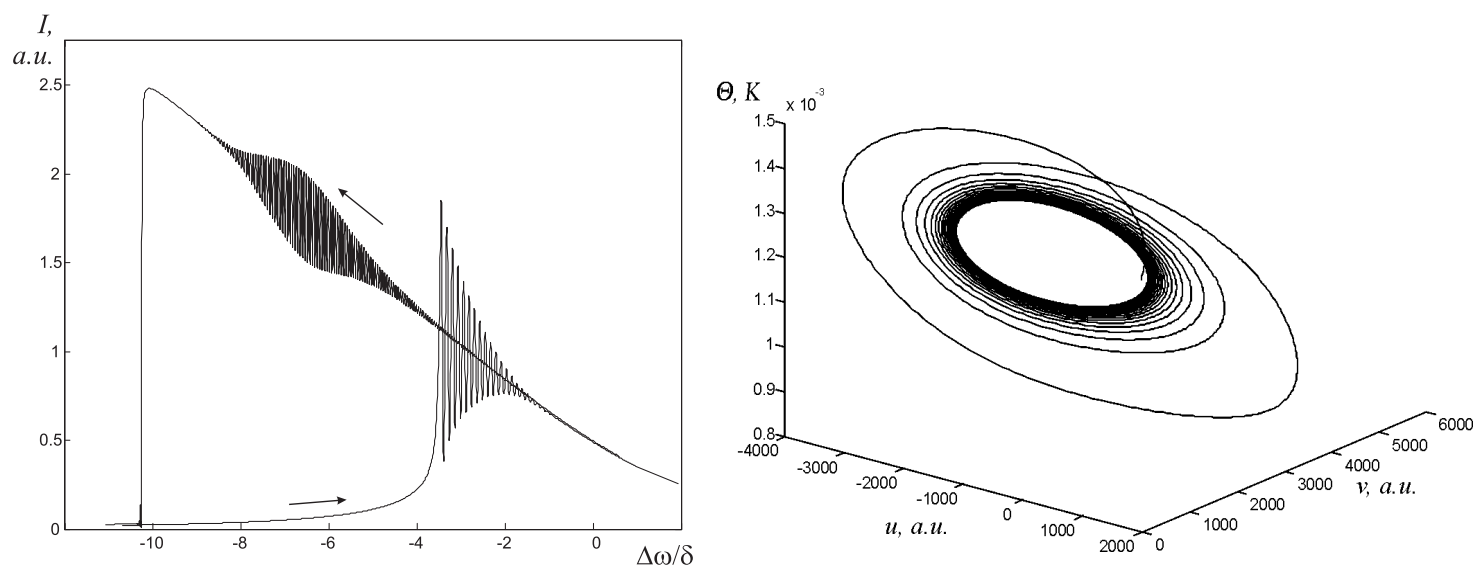

Figure 3. Numerical simulation of WGM nonlinear optical resonance in microspheres with thermal nonlinearity. Hysteretic response curve (left) and phase diagram (right). In both optical and numerical experiments, the oscillatory behavior was observed on both branches of response curves.

Numerical experiments have also shown that when Kerr nonlinearity obeys condition (22), oscillations are not observed and ultimate cycle again turns to stable focus. In experiments, however, thermal oscillations dynamics sometimes adopted chaos-resembling character. This behavior may be explained, as numerical modelling confirms, if model of two or more neighboring optical modes is applied. In real microspheres each mode is a doublet due to internal backscattering, which leads to sumultaneous existence of two counter-propagating modes. ${ }^{8}$ In this system orbital cycle can become unstable (Fig.4).

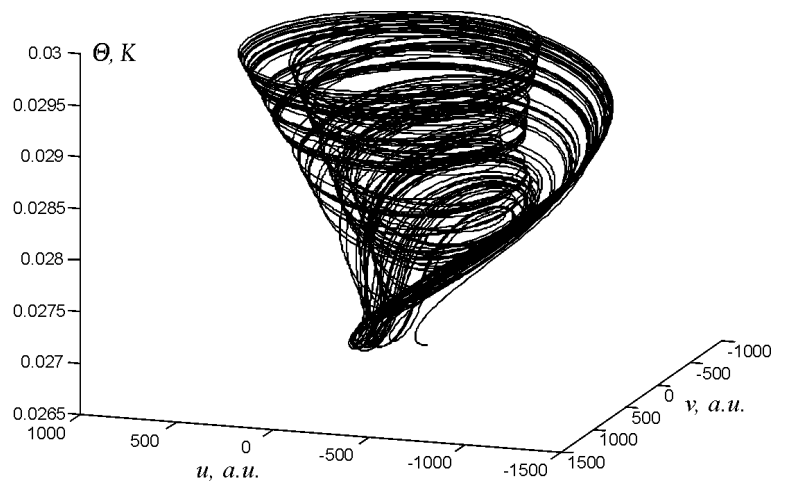

Figure 4. Numerical simulation of chaotic regime in microsphere with thermal nonlinearity and two close optical modes

\section{EXPERIMENTAL OBSERVATION OF THE OSCILLATIONS}

To observe the oscillations, we used small $2 R \simeq 25 \div 160 \mu \mathrm{m}$ fused silica microspheres with quality factors of the order of $Q \simeq 10^{7} \div 10^{8}$, manufactured with the hydrogen-oxygen mini-torch. In order to avoid degradation of $Q^{-}$ factor due to adsorption of atmospheric water, microspheres were placed upon fabrication into the special chamber where all measurements were carried out. The chamber was filled with dry clean nitrogen. Whispering gallery modes were excited in microspheres with a prism coupler, using $\mathrm{He}-\mathrm{Ne}$ laser $(\lambda=0.63 \mu \mathrm{m})$ with piezo-driven 


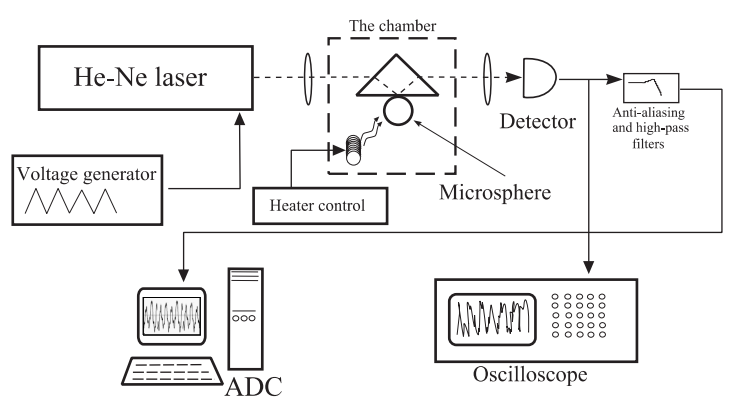

Figure 5. The scheme of experimental setup.

front mirror. Laser frequency could be tuned within the range of approximately $0.7 G H z$. Laser's maximum output power was about $540 \mu \mathrm{W}$. To measure the quality-factor, the power fed into the microspheres was decreased with neutral glass filters to weaken thermal and Kerr nonlinearities.

An incandescent tungsten filament heater was placed near the resonator and used for coarse tuning of resonator eigenfrequency in the range of about $30 \mathrm{GHz}$. Narrow-range laser frequency sweeping (in the range of $700 \mathrm{M} \mathrm{Hz}$ ) was carried out by applying ramp voltage to the piezo-actuator of laser mirror. This voltage produced sweeping of the light frequency and allowed to observe resonant curves on the screen of the oscilloscope. The signal from detector was observed with digital oscilloscope, or sampled with ADC computer board.

In this work, the intentional search and observation of oscillations in the WGM was carried out for the first time. Instead of using high-purity fiber-grade sol-gel glasses similar to those used in the majority of recent experiments, we fabricated microspheres out of original technical grade (96\%) silica which possessed increased absorption and yielded pronounced thermal nonlinearity of the WGMs. Low grade of this silica was confirmed by reduced melting temperature, and its increased luminance in molten state.

Fig.6 depicts thermorefractive oscillations observed in the microsphere with the diameter $82.5 \mu \mathrm{m}$. This picture presents superimposed resonant curves obtained by scanning the laser in two opposite directions in the vicinity of a whispering-gallery mode. For this, triangular ramp voltage was applied to the piezo actuator of the laser mirror. The hysteretic feature due to nonlinearity can easily be observed, as well as thermal oscillations on the slope of nonlinear resonant curve. The curve is slightly tilted due to the high-pass filter used in the setup to get rid of constant offset. The oscillating mode has indices $l=2 \pi R n / \lambda \simeq 600, l-m=6 \pm 2$ as it was calculated from observations of the WGM's belt. This belt is caused by scattering of light on surface roughness and dust of the microsphere. Oscillations were observed with $540 \mu \mathrm{W}$ of pump power incoming on the objective lens preceding the chamber with the microsphere. The measured $Q$-factor of the mode, inferred at reduced pump power in "linear" regime, was $(6-7) \times 10^{8}$. The oscillations observed with the sweep turned off were close to harmonic ones with dominating spectral peak at $26 \mathrm{kHz}$ accompanied by numerous smaller harmonics and the background of continuous spectrum.

In the microsphere with diameter of $90 \mu \mathrm{m}$ maximal coupling provided for $30 \%$ of $540 \mu \mathrm{W}$ of pump power to be consumed by the WGM. The oscillations were observed down to $20 \mu \mathrm{W}$ (4\%) of coupling and disappeared when coupling was reduced to consume about $10 \mu \mathrm{W}$. They also disappear with the decrease of Q-factor (which may happen due to gradual contamination of the microsphere's surface) and with decrease of gap between the microsphere and the prism coupler (decrease of loaded Q). Characteristic frequency of these oscillations was 7 $\mathrm{kHz}$ with harmonics at 14 and $21 \mathrm{kHz}$. The spectrum was continuous and width of these harmonics was of the order of kilohertz. All these effects were reproducible in our numerical model with approximately the same set of parameters as in experiment. We identify this oscillatory behavior mainly as the result of nonstationary and chaotic effects in the system with more than one optical mode (usually doublets) in presence of thermal nonlinearity. This possibility was confirmed by numerical simulations (see section V). Direct quantitative comparison, however, was not possible due to some uncertainty in the estimate of the actual power absorbed in the resonator and the value of coefficient of absorption which could not be measured directly in our experiments as the quality-factor provides only the value of the sum of internal scattering, absorption and surface losses. 

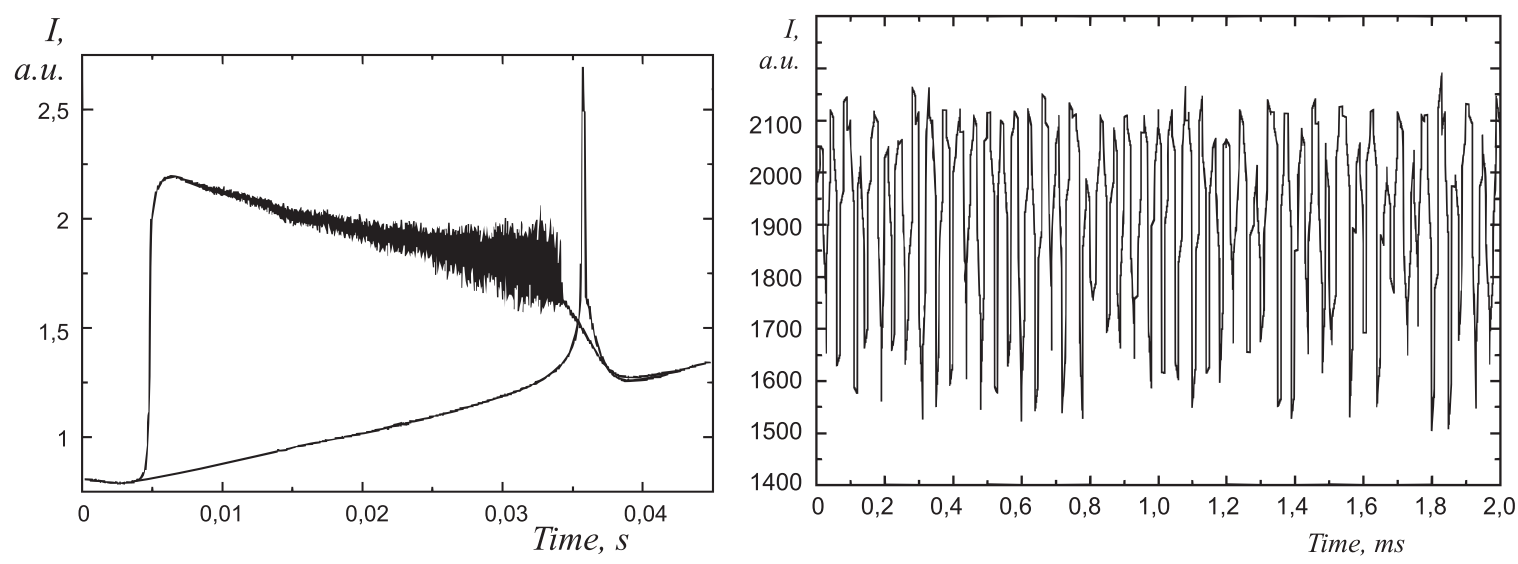

Figure 6. Thermal nonlinearity and thermal oscillations in the microsphere with the diameter $82.5 \mu \mathrm{m}$. Sweep rate is $2.9 \mathrm{GHz} / \mathrm{s}$. Right picture represents thermal oscillations on the slope of the nonlinear resonant curve shown to the left

The onset of oscillations in this resonator was preceded by a specific nonstationary process. This process emerges every time when the nonlinearity is strong and power consumption in a mode is high enough. The process can be observed when the frequency sweep is on, and one can see the dynamically refreshing resonant curve on the screen of the oscilloscope. Typical parameters accompanying the onset of the process are about $100 \mu W$ of power consumption in the mode, $Q \simeq 10^{8}$ and nonlinearity-induced widening of the resonant curve is above $100 \mathrm{MHz}$. The process itself looks like splitting of WGM, or in other words, emerging of satellite resonant frequencies which change dynamically and irreversibly. Initially, the ordinary highly nonlinear resonant curve can be seen on the screen. Then, from the point of the greatest energy consumption the second peak emerges, then another. These peaks are moving "down the resonant curve", there can be several such moving peaks. Sometimes, the oscillations emerge with one of these peaks and the oscillating area also move "down the slope" until it stabilizes on the slope of the resonant curve. This sequence is represented on the following series of Fig. 7 , where one can see the superimposed resonant curves obtained by scanning the laser frequency in two opposite directions in the vicinity of the WGM. The whole process takes about few minutes or less, the time scale depends on the optical pump power.
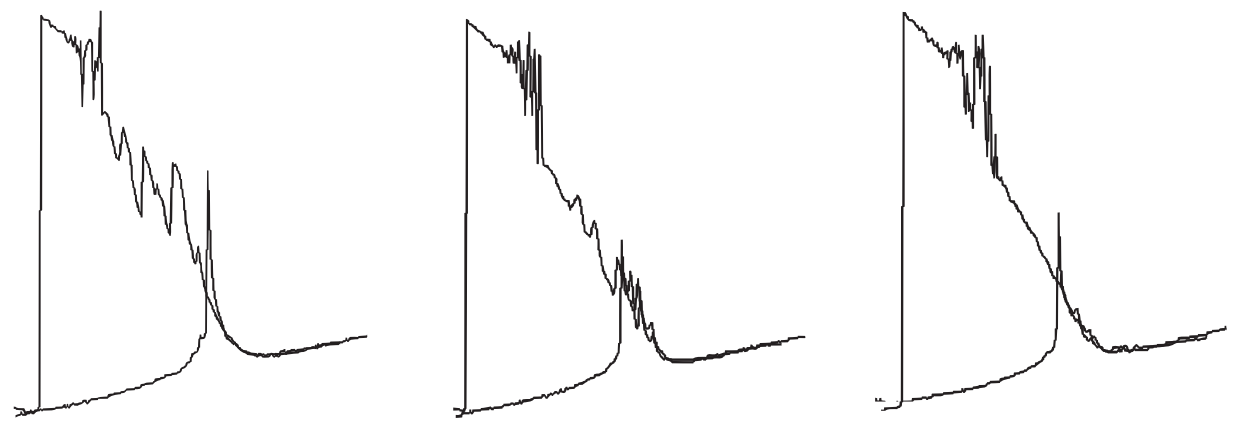

Figure 7. Nonstationary thermal effects and bifurcations in the microsphere with the diameter $90 \mu \mathrm{m}, Q \simeq 10^{8}$. The oscillating area is to the top of the curve. Several accompanying peaks on the picture to the left are moving "down the resonant curve" and disappear later as seen on the picture to the right.

\section{CONCLUSION}

We have presented the theoretical analysis of the effects in microspheres associated with concurrence of Kerr and thermal nonlinearities. It can be seen that the main features of the experimentally observed oscillations 
are well captured by the analysis. However, experimentally observed effect is even more complex and requires additional explanations. We have observed inconclusive evidence of small but significant irreversible changes of the mode quality factors in the case of the oscillatory process under consideration. These changes also lead to degradation of oscillations when certain power threshold is reached. When the oscillations were observed and digitized without the frequency sweep, they disappeared within 15 seconds and when the frequency sweep was restored we found three new modes (triplet) in place of original mode. After some minutes, oscillations resurrected and these three modes merged into a single nonlinear mode but its Q-factor dropped significantly (about 2-5 times). We may suggest that the process of oscillation causes some degradation of material. The absorption of optical power may be not homogeneous inside contaminated fused silica but connected with small loss centers. Large thermal pulsations of these heated centers with relatively high frequency could produce defects in the material of the microsphere analogous to "hole burning" which lead to increased losses due to additional scattering. These local defects may partially restore after some time. Collapse and channelling of WGM due to self-focusing produced by thermal nonlinearity again accompanied by large local heating and appearance of defects may be also considered. The described nonstationary and irreversible effects have also been observed in experiments with other microspheres. However, the detailed study of the phenomena should be subject of separate experiments that go beyond the scope of current paper.

Summing up, we observe experimentally and describe qualitatively and quantitatively an interesting effect of thermal oscillations and bifurcations in optical microspheres as well as unusual irreversible processes at microwatt level of optical power. These effects, that may be undesirable in future applications of microspheres, happily disappear if modern high-grade quality materials are used.

\section{ACKNOWLEDGMENTS}

This work was supported by the President of Russian Federation grant for young scientists \#MD-208.2003.02 and grant \#NS-1318.2003.2.

\section{REFERENCES}

1. V. Braginsky, M. Gorodetsky, and V. Ilchenko, "Quality-factor and nonlinear properties of optical whispering-gallery modes," Phys. Lett. A137, pp. 393-397, 1989.

2. V. Ilchenko, M. Gorodetsky, X. Yao, and L. Maleki, "Microtorus: a high-finesse microcavity with whisperinggallery modes," Opt. Lett. 26, pp. 256-258, 2001.

3. D. Armani, T. Kippenberg, S. Spillane, and K. Vahala, "Ultra-high-q toroid microcavity on a chip," Nature 421, pp. 925-928, 2003.

4. M. Gorodetsky and V. Ilchenko, "Thermal nonlinear effects in optical whispering-gallery microresonators," Laser Phys. 2, pp. 1004-1009, 1992.

5. A. Salomonovich, "Introduction to bayesian image analysis," Zhurnal Tekhnicheskoi Fiziki (in russian) 22, p. $245,1952$.

6. G. Belokopytov Vest. Mosk. Univer. (in russian) Ser.3, Fiz. Astron.,\#3, 11, 1997.

7. M. Gorodetsky, A. Savchenkov, and V. Ilchenko, "On the ultimate q of optical microsphere resonators," Opt. Lett. 21, pp. 453-455, 1996.

8. M. Gorodetsky, A. Pryamikov, and V. Ilchenko J. Opt. Soc. Am. B. 17, pp. 1051-1057, 2000.

9. M. Lines, "Scattering losses in optic fiber materials. i. a new parametrization. ii. numerical estimates," $J$. Appl. Phys. 55, pp. 4052-4063, 1984. 